\title{
Superconducting SET with tunable electromagnetic environment
}

\author{
Michio Watanabe ${ }^{1}$, Koji Ishibashi, Yoshinobu Aoyagi \\ Semiconductors Laboratory, RIKEN, and CREST-JST, 2-1 Hirosawa, Wako-shi, Saitama 351-0198, Japan
}

\begin{abstract}
We have studied the environmental effect on superconducting single-electron transistors (S-SETs) by biasing SSETs with arrays of small-capacitance dc SQUIDs, whose effective impedance can be varied in situ. As the zerobias resistance of the arrays is increased, Coulomb blockade in the S-SET becomes sharper, and the gate-voltage dependence changes from $e$-periodic to $2 e$-periodic. The SQUID arrays could be used as on-chip noise filters.
\end{abstract}

Key words: Small-capacitance Josephson junction; Single-electron transistor; Electromagnetic environment; Coulomb blockade

\section{Introduction}

Small-capacitance Josephson junction has been playing an important role in studying the interplay between quantum mechanically conjugate valuables, the Josephson phase and the charge on the island electrode [1]. It is also a promising candidate for solidstate realizations of quantum computing [2]. In this system, coupling to an electromagnetic environment is a key issue. The coupling influences the interplay between the phase and the charge. Moreover, it is a major source of decoherence in quantum bits.

In this work we study the environmental effect on superconducting single-electron transistors (S-SETs), which are building blocks of nano-circuits. The effects of dissipation on S-SETs have been investigated by capacitively coupling a two-dimensional electron gas to an S-SET [3]. We take a different approach as described in the next section.

\section{Experiment}

We have biased S-SETs with one-dimensional (1D) arrays of small-capacitance dc superconducting quantum interference devices (SQUIDs) as shown in Fig. 1. With the SQUID configuration, the effective impedance of the arrays can be varied in situ by applying a weak $(1-10 \mathrm{mT})$ external magnetic field $B$, i.e., the arrays are tunable environment $[4,5,6]$ for the SET. For a scanning electron micrograph of a SQUID

\footnotetext{
1 Corresponding author. E-mail: michio@postman.riken.go.jp
}

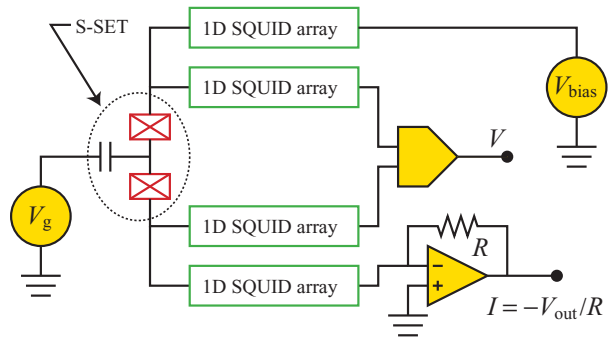

Fig. 1. Schematic diagram of the sample and the circuit.

array, see Fig. 1 of Ref. [6]. We measured the SET in a four-point configuration, where the bias was applied through one pair of SQUID leads and the potential difference was probed through the other pair of leads. The SQUID arrays could be measured in a two-point configuration on the same side of the SET, and the zero-bias resistance $R_{0}^{\prime}$ obtained in this measurement may characterize the environment [4].

The samples consist of $\mathrm{Al} / \mathrm{Al}_{2} \mathrm{O}_{3} / \mathrm{Al}$ junctions fabricated on $\mathrm{SiO}_{2} / \mathrm{Si}$ substrate. The area of each junction in the SET is $0.1 \times 0.1 \mu \mathrm{m}^{2}$, so that the charging energy of the SET is on the order of $1 \mathrm{~K}$.

\section{Results and discussion}

We show in Fig. 2 low-temperature $(T=0.02 \mathrm{~K})$ current-voltage $(I-V)$ curves of an S-SET in different environments, $R_{0}^{\prime}=0.2 \mathrm{M} \Omega(B=0)$ for a and $R_{0}^{\prime}=$ $0.3 \mathrm{G} \Omega(B=6.8 \mathrm{mT})$ for b. Here, the temperature is $0.02 \mathrm{~K}$ and the gate voltage, $V_{\mathrm{g}}$, is 0 . For the sam- 


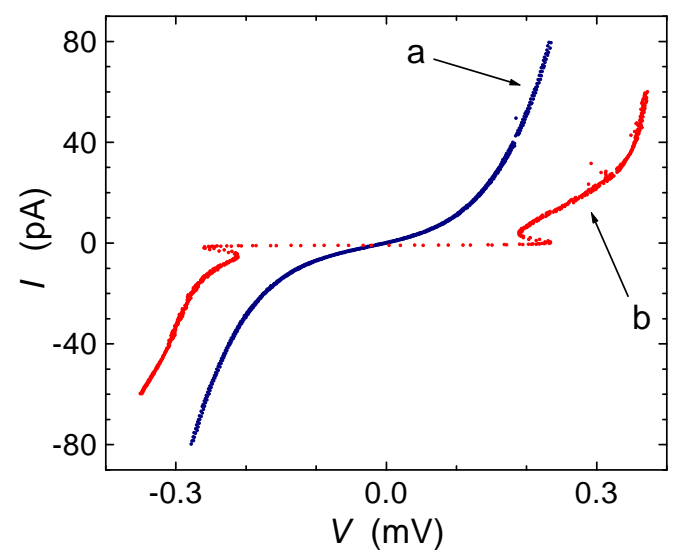

Fig. 2. Current-voltage characteristics of a superconducting SET at $T=0.02 \mathrm{~K}$ in different environments.

ple in Fig. 2, the normal-state resistance of the SET is $0.10 \mathrm{M} \Omega$, so that the Josephson energy is estimated to be $0.1 \mathrm{~K}$. The parameters of the SET should be the same for the two curves in Fig. 2, because the SET does not have a SQUID configuration and the field applied here is much smaller than the critical field for Al films $(\approx 0.1 \mathrm{~T})$. The environment for the SET (the SQUID arrays), however, is strongly varied with $B$. The behavior of the SET demonstrated in Fig. 2 does not result from the magnetic-field influencing the $I-V$ curve of the SET, but rather from an environmental effect on the SET. While Coulomb blockade in curve a is rounded, that in curve $b$ is sharp. Furthermore, curve $b$ has a 'back-bending' in the low-current part, which is evidence of coherent single-Cooper-pair tunneling [5]. For the difference between curves a and b quasiparticles generated by external noise may be responsible, because no filter has been installed in or at the top of our cryostat. A sufficiently large $R_{0}^{\prime}$ would weaken the coupling to the noise source and suppresses the quasiparticle generation.

We have also examined the dependence on $V_{\mathrm{g}}$ as shown in Fig. 3. We fixed $V_{\text {bias }}$ (see Fig. 1) and measured the current $I$ as a function of $V_{\mathrm{g}}$. When $V$, the the potential difference across the SET is large compared to the voltage drop at the other parts of the circuit, $V$ is nearly independent of $V_{\mathrm{g}}$. This is the case for the upper data set in Fig. $3\left(R_{0}^{\prime}=0.2 \mathrm{M} \Omega, B=0\right)$, and $V=0.1 \mathrm{mV}$. In the opposite case, $V$ also oscillates as a function of $V_{\mathrm{g}}$, which makes the amplitude of the current oscillation small. In the case of the lower data set in Fig. $3\left(R_{0}^{\prime}=0.3 \mathrm{G} \Omega, B=6.8 \mathrm{mT}\right), V$ oscillates between 0.1 and $0.3 \mathrm{mV}$. Nevertheless, it is still possible to clearly observe the oscillation of $I$. In Fig. $3, V_{\mathrm{g}}$ is normalized by $e / C_{\mathrm{g}}$, where the gate capacitance $C_{\mathrm{g}}=6 \mathrm{aF}$ is estimated from a similar gatesweep measurement in a strong enough magnetic field $(B=0.1 \mathrm{~T})$ to drive the sample into the normal state.

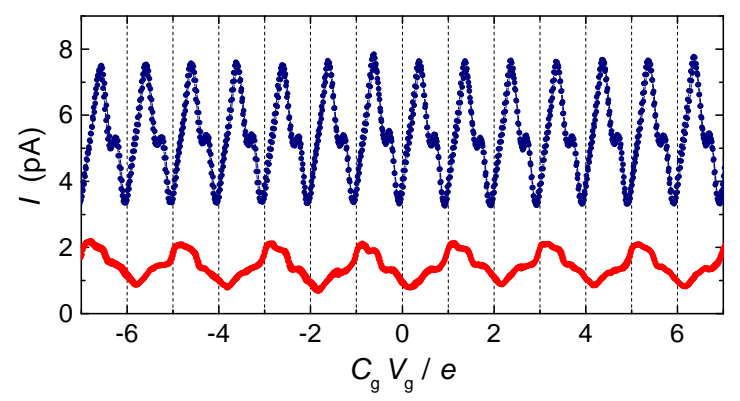

Fig. 3. Current vs. normalized gate voltage at $T=0.02 \mathrm{~K}$ for the same superconducting SET as in Fig. 2. The upper (lower) data set was taken in the same environment as for curve a (b) in Fig. 2.

By tuning the environment, the $V_{\mathrm{g}}$ dependence changes from $e$-periodic to $2 e$-periodic, i.e., the dominant tunneling process changes from quasiparticle tunneling to Cooper-pair tunneling. This observation is consistent with the picture in the end of the preceding paragraph that the quasiparticle generation is suppressed by the SQUID arrays when the arrays have a sufficiently large $R_{0}^{\prime}$.

\section{Conclusion}

We could sharpen Coulomb blockade in the S-SET and change the dependence on the gate voltage from $e$ periodic to $2 e$-periodic, by tuning the electromagnetic environment composed of small-capacitance SQUID arrays in the immediate vicinity of an S-SET. The SQUID arrays behaved as effective noise filters.

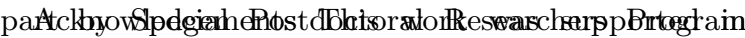
and President's Special Research Grant of RIKEN.

References

[1] G. Schön, A. D. Zaikin, Phys. Rep. 198 (1990) 237; S. L. Sondhi, S. M. Girvin, J. P. Carini, D. Shahar, Rev. Mod. Phys. 69 (1997) 315.

[2] Y. Nakamura, Yu. A. Pashkin, J. S. Tsai, Physica B 280 (2000) 405; for recent progress in Josephson-junction quantum bits, see, e.g., R. Fitzgerald, Phys. Today 55 (2002) No. 6, p. 14.

[3] J. B. Kycia, J. Chen, R. Therrien, C. Kurdak, K. L. Campman, A. C. Gossard, J. Clarke, Phys_Rev_Lett. 87 (2001) 017002; A. J. Rimberg, W. Lu, cond-mat/0205382 (2002).

[4] M. Watanabe, D. B. Haviland, Phys. Rev. Lett. 86 (2001) 5120.

[5] M. Watanabe, D. B. Haviland, R. L. Kautz, Supercond. Sci. Technol. 14 (2001) 870. 
[6] M. Watanabe, D. B. Haviland, J. Phys. Chem. Solids 63 (2002) 1307. 